\title{
The Impact of Simulated Weightlessness on Endothelium-Dependent Angiogenesis and the Role of Caveolae/Caveolin-1
}

\author{
Fei Shi ${ }^{a}$ Tian-Zhi Zhao ${ }^{b}$ Yong-Chun Wang ${ }^{a}$ Xin-Sheng Cao ${ }^{a}$ Chang-Bin Yang ${ }^{a}$ \\ Yuan Gao ${ }^{a}$ Cheng-Fei Lia Jiang-Dong Zhao ${ }^{a}$ Shu Zhanga Xi-Qing Sun ${ }^{a}$ \\ aDepartment of Aerospace Biodynamics, Faculty of Aerospace Medicine, The Fourth Military Medical \\ University, Xi'an, b Department of Neurosurgery, Tangdu Hospital, The Fourth Military Medical \\ University, Xi'an, China
}

\section{Key Words}

Simulated microgravity $\bullet$ Endothelial cells $\bullet$ Angiogenesis $\bullet$ Caveolin-1 $\bullet$ eNOS

\begin{abstract}
Background/Aims: The potential role of caveolin-1 in modulating angiogenesis in microgravity environment is unexplored. Methods: Using simulated microgravity by clinostat, we measured the expressions and interactions of caveolin-1 and eNOS in human umbilical vein endothelial cells. Results: We found that decreased caveolin-1 expression is associated with increased expression and phosphorylation levels of eNOS in endothelial cells stimulated by microgravity, which causes a dissociation of eNOS from caveolin-1 complexes. As a result, microgravity induces cell migration and tube formation in endothelial cell in vitro that depends on the regulations of caveolin-1. Conclusion: Our study provides insight for the important endothelial functions in altered gravitational environments.
\end{abstract}

\section{Introduction}

In space missions the human body undergoes a series of pathophysiological changes, such as bone loss, muscle atrophy and impaired immunity [1-5]. In particular, hydrodynamic alterations in cardiovascular systems induced by microgravity are tightly associated with orthostatic intolerance following space flight [4, 6-8]. Since endothelial cells are key players in maintaining vascular integrity, modulating immune response and participating in vessel

F. Shi, T.-Z. Zhao and Y.-C. Wang contributed equally to this work.

Shu Zhang, MD, PhD and Xi-Qing Sun, MD, PhD 
remodeling, the effects of weightlessness on endothelial cells have been extensively studied $[9,10]$. However, the important aspects of the underlying mechanisms still remain unclear.

Angiogenesis, a form of neovascularization from the existing blood vessels, is distinct from vasculogenesis or arteriogenesis $[11,12]$. As an important structural adaptation to increase vascular perfusion, angiogenesis requires coordinated processes in endothelial cells that include vasodilation, sprouting, cell proliferation, migration and maturation $[11,12]$. These angiogenic processes require production of nitric oxide (NO) from activated endothelial nitric oxide synthase (eNOS), which is disrupted in a variety of vascular disorders [13]. In both pathological and physiological scenarios $[14,15]$, eNOS-dependent angiogenesis can be promoted by the increased expression levels or enhanced phosphorylation at its C-terminus angiogenic factors such as vascular endothelial growth factor (VEGF). In addition, it has been established that subcellular localization and/or associated chaperons regulate eNOS function [16-19], further changing the angiogenic potential in endothelial cells. In vitro angiogenesis can be evaluated by migration and tube formation assays, both of which have been successfully performed using human umbilical vein endothelial cells (HUVEC) [20]. In fact, the effects of microgravity on angiogenesis have been studied in HUVEC, in which the in vitro formation of capillary-like tubes on a 3D-matrix culture system was assessed [21]. During this study, simulated weightlessness was shown to increase the tube formation capacity of HUVEC, which was dependent on NO production [21]. It was also found that PI3K/ Akt-stimulated eNOS phosphorylation acts upstream of eNOS activation by microgravity in endothelial cells, while Cav-1-mediated mechanisms could be involved as well [21].

Caveolin-1 is the major scaffolding protein for organizing the unique membrane invaginations-caveolae [22]. Particularly abundant in the endothelium, caveolae exist as 50$100 \mathrm{~nm}$ microdomains mainly in the plasma membrane that can be observed by electron microscopy. Not only as nonclathrin-coated vesicles for the transcytosis of molecules across end othelial cells, caveolae are also important signaling platforms [23, 24]. For instance, eNOS is targeted in caveolae for its activation $[16,25,26]$, and Cav-1 plays an inhibitory effect through direct protein binding with a specific domain of eNOS [16]. It has been documented that decreasing Cav-1 association with eNOS protein results in the eNOS enzyme activation $[17,27,28]$.

Given the potential involvement of Cav-1 in mediating eNOS activation by microgravitystimulated angiogenesis, Cav-1 may play a role in angiogenesis stimulated by weightlessness. By dissecting the functions of caveolae/Cav-1 in microgravity-induced endothelial cell functions including cell migration and tube formation, our data provide a novel mechanistic insight for the molecular pathways underlying the promotion of angiogenesis by microgravity.

\section{Methods and Materials}

\section{Cell culture}

HUVEC were purchased from Lonza, then were subcultured and propagated in EGM-2 medium (Lonza, USA) at $37^{\circ} \mathrm{C}, 5 \% \mathrm{CO}_{2}$. The cells with passage numbers less than 7 were used in the experiments.

\section{Simulated microgravity}

A clinostat-based strategy was used to simulate weightlessness in endothelial cells [21]. In brief, HUVEC cells were grown on gelatin-coated coverslips, and then incubated in vessel chambers filled with culture medium. Cells were mainly separated into two groups that exposed to either normal gravity (NG, kept static), or simulated microgravity (Clino, clinorotation at $30 \mathrm{rpm}$ ) both for 24 hours at humidified incubator at $37^{\circ} \mathrm{C}, 5 \% \mathrm{CO}_{2}$.

\section{Immunofluorescence and epifluorescence microscopy}

The cells were fixed with paraformaldehyde followed by permeabilization with $0.2 \%$ Triton-X in PBS, and then blocked with 1\% BSA for 1 hour. A primary rabbit polyclonal anti-Cav-1 antibody (Abcam, USA), and a secondary goat anti-rabbit antibody conjugated with Alexa Fluor 594 were used. For a negative

\section{KARGER}




\section{Cellular Physiology Cell Physiol Biochem 2016;38:502-513

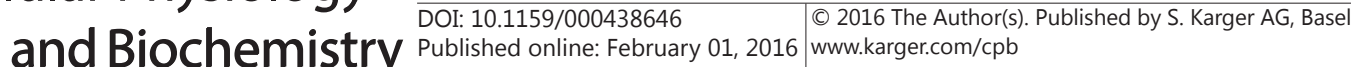

Shi et al.: Endothelial Functions in Altered Gravitational Environments

control, identical cells were exposed to the secondary antibody alone. Before imaging, the cell nuclei were counter stained by DAPI in Vectashield mounting medium (Vector Labs, USA). Epifluorescence signals were then imaged by Olympus inverted fluorescent microscope, and then processed by Image J from NIH.

\section{Co-immunoprecipitation and Western blotting}

After the treatments, endothelial cells on the slides were washed twice with PBS and then lysed in RIPA buffers on ice. After protein measurements, the same amounts of protein extracts were immunoprecipitated by anti-Cav-1 (Abcam, USA) and protein A-sepharose beads at $4^{\circ} \mathrm{C}$ overnight. The immunoprecipitated beadsassociated complexes were then washed 4 times by RIPA buffer and boiled in $2 \times$ laemmli sample buffer for 10 min. The proteins in the supernatants were then resolved in PAGE followed by Western blotting. The anti-eNOS, anti-phosphorylated eNOS (Ser1177) and anti-Cav-1 antibodies (Abcam, USA) were used for blotting the proteins, respectively. The aliquots of samples before immunoprecipitation were also blotted as the input controls. Anti-GAPDH (Abcam, USA) was used for loading control.

\section{Cav-1 RNAi knockdown and overexpression}

The knockdown of Cav-1 in HUVEC cells were performed by a commercial available siRNA-mediated strategy (Santa Cruz, USA). The siRNA reagents that consist of pools of three to five Cav-1-specific 19-25 nt siRNAs (sc-29241, Santa Cruz, USA) were transfected into endothelial cells by Lipofectamin 2000 (Invitrogen, USA). The control knockdown used a scrambled sequence (Control siRNA-A, sc-37007, Santa Cruz, USA) in the transfection. For the overexpression of Cav-1 in HUVEC cells, human Cav-1 (ORF (NM_001753) was cloned into pCMV2 mammalian expression vector, while empty pCMV2 vector was used as control. The plasmids were transfected in cells using Nucleofector (Lonza, USA).

\section{Quantitative PCR}

Gene expressions were measured by quantitative PCR for the endothelial cells harvested after the treatments. Total RNA was extracted by Trizol (Invitrogen, USA) and reverse transcribed into cDNA by using kits (Applied Biosystems, USA). The qPCR primers used included: Cav-1 (NM_001753.4, forward: 5'-ACC CAC TCT TTG AAG CTG TTG-3', reverse: 5'-AAC TTG AAA TTG GCA CCA GG-3', 138 bp); eNOS (NM_000603.4, forward: 5'-TAG CTG TGC TGG CAT ACA GG-3', reverse: 5'-ATG GTC AAG TTG GGA GCA TC-3', 296 bp); GAPDH (NM_002046.4, forward: 5'-AAC GGA TTT GGT CGT ATT G-3', reverse: 5'-GGA AGA TGG TGA TGG GAT T-3', $208 \mathrm{bp}$ ). The PCR reactions were performed by cyber green-based system (Applied Biosystems, USA), and the expression levels were normalized to GAPDH mRNA.

\section{Cell migration assay}

After the treatments, a scratch assay was performed to assess the ability of cell migration [21]. In brief, a wound was generated by scraping confluent cells with a 200 ul pipette tip, which was marked as the cell boundary at time zero. 24 hours later, the migrating cells into the scratches were monitored in an Olympus microscope, and the distance between the front edges of migrating cells was measured and averaged. The results of migration were normalized to the cells exposed to the normal gravity.

\section{Tube formation assay}

The in vitro tube formation in HUVEC cells was performed in Matrigel as previously described [21]. Briefly, Matrigel matrix (BD bioscience, USA) was thawed in ice overnight and then prepared in 24-well plate $(250 \mu \mathrm{l})$ at $37^{\circ} \mathrm{C}, 5 \% \mathrm{CO}_{2}$ for 1 hour. After the treatments, endothelial cells on the slides were trypsinized and cell numbers were counted. Then $400 \mu \mathrm{l}$ cells at $2 \times 10^{5} / \mathrm{ml}$ were seeded onto the pre-solidified matrigel in the wells with $10 \%$ fetal bovine serum in the medium. Tubes formed 20 hours later were imaged with a microscope, and quantified by the measurement of the tube length in five random areas in Image J from $\mathrm{NIH}$. Experiments were repeated at the least three times and the results were calculated as the relative fold to the control treated cells.

\section{Statistical Analysis}

Data were presented by mean \pm SEM from at least three independent experiments. Statistical comparisons between groups were performed by one-way ANOVA followed by Student's t-test with significances at $* \mathrm{p}<0.05$ for the comparisons as indicated. 


\section{Results}

Simulated weightlessness attenuates caveolin-1 in HUVEC cells

It has been shown that both the structural integrity and the distribution density of caveolae in the plasma membrane of HUVEC cells were attenuated in response to simulated microgravity [21]. Notably, we also found the similar changes in our experiments that examined Cav-1 protein localization using immunofluorescence microscopy (Fig. 1A). Although it is rather difficult for quantitative imaging-based measurements, it appears that Cav-1 signal decreased in cells exposed to microgravity, compared to normal gravity controls. Consistent with this hypothesis, quantitative PCR measurements showed that there was a significant decrease (more than 50\%) of Cav-1 mRNA (Fig. 1B) in microgravity-treated cells, comparing to the controls. In order to confirm the changes in gene expression, we also used Western blotting to examine the changes of Cav-1 at the protein level. As shown in Fig. 1C and 1D, following exposure to weightlessness for 24 hours, Cav-1 protein also decreased in HUVEC cells.

\section{Increased eNOS expression/phosphorylation in HUVEC cells exposed to microgravity}

As an important vascular signaling protein that associates with Cav-1, we then measured eNOS expression (Fig. 2). Comparing to the controls, we found an approximately two-fold increase of eNOS mRNA levels in microgravity-treated groups by quantitative PCR (Fig. 2A). In Western blot, the levels of both total protein and phosphorylated form of eNOS were enhanced in cells exposed to microgravity (Fig. 2B and 2C). These data confirmed the potential implications of eNOS activation in endothelial cells stimulated by weightlessness, which was consistent with the previously published findings [21].

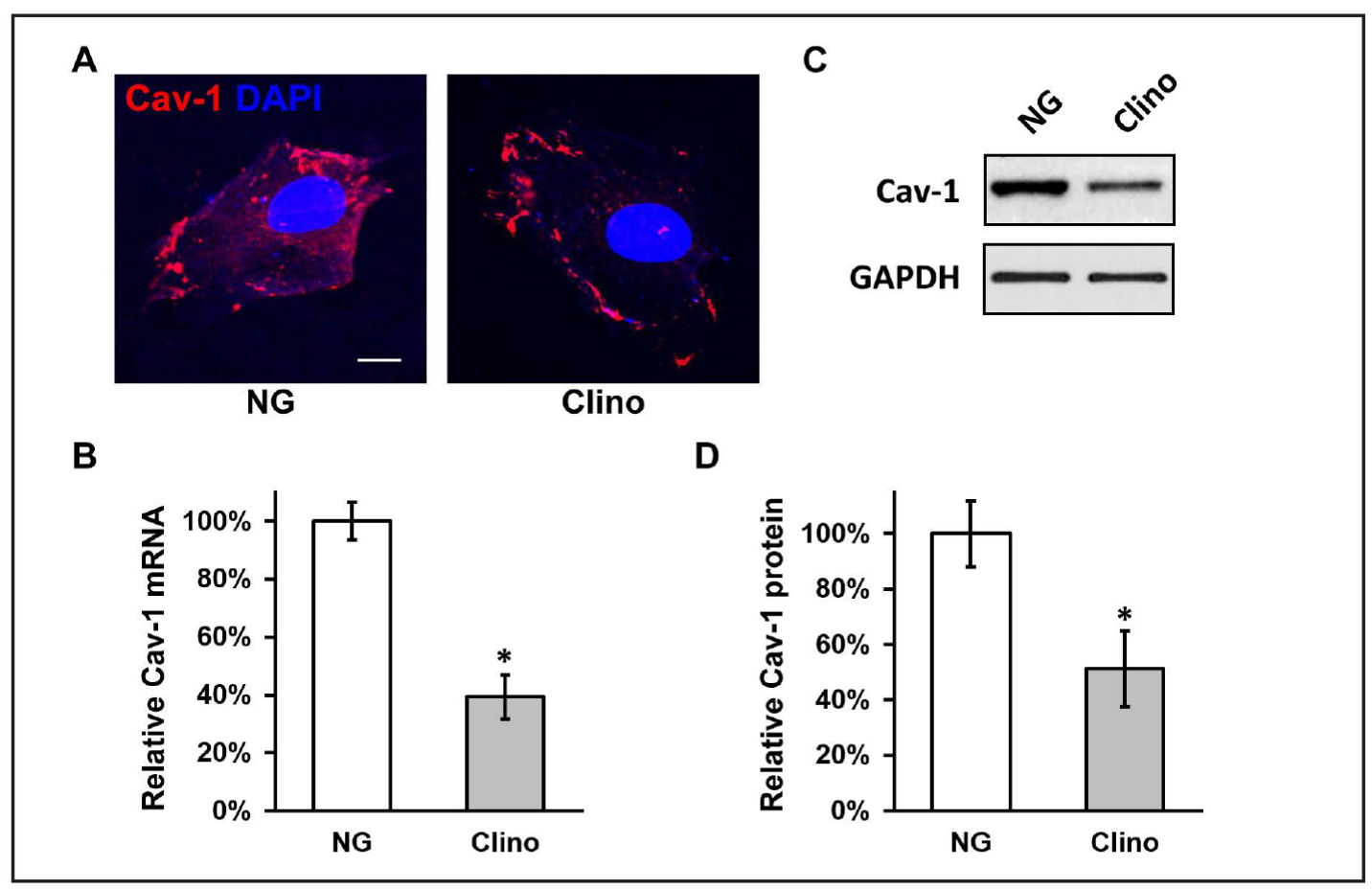

Fig. 1. Effects of simulated microgravity on Cav-1 localization and expression in HUVEC-C. (A) Confocal microscopy localization of Cav-1 (red) in HUVEC-C exposed to $24 \mathrm{~h}$ of normal gravity (NG) or simulated microgravity (Clino). Nucleus is labelled with DAPI in blue. Scale bar $10 \mu \mathrm{m}$. (B to D) Levels of mRNA (B) and protein (C and D) of Cav-1 in HUVEC-C exposed to $24 \mathrm{~h}$ of normal gravity (NG) or simulated microgravity (Clino). mRNA levels were normalized to $G A P D H$, and plotted as percentage relative to NG group. (D) Western blots were quantified by densitometry analysis. Values are mean \pm SEM from three independent experiments. * $\mathrm{P}<0.05$ compared to NG. 


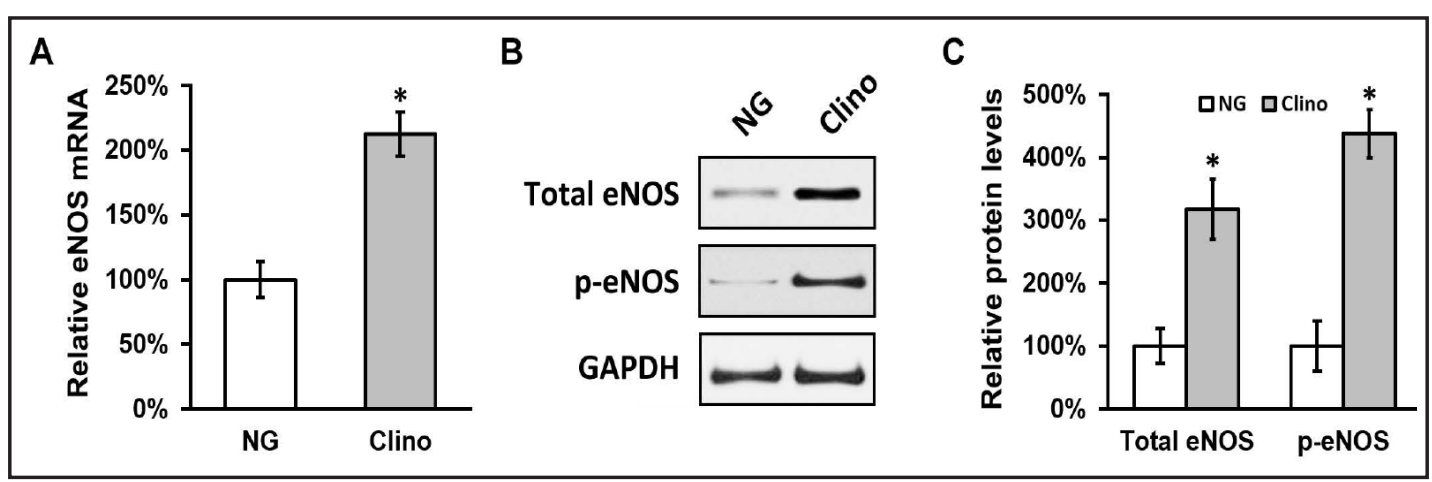

Fig. 2. Effects of simulated microgravity on eNOS expression and phosphorylation in HUVEC-C. (A) Levels of eNOS mRNA were examined in HUVEC-C exposed to $24 \mathrm{~h}$ of normal gravity (NG) or simulated microgravity (Clino). mRNA levels were normalized to GAPDH, and plotted as percentage relative to NG group. (B) Total and phosphorylated eNOS protein levels were examined in HUVEC-C exposed to $24 \mathrm{~h}$ of normal gravity (NG) or simulated microgravity (Clino). (D) Western blots were quantified by densitometry analysis. Values are mean \pm SEM from three independent experiments. ${ }^{*} \mathrm{P}<0.05$ compared to NG.

Fig. 3. Effects of simulated microgravity on the interaction between Cav-1 and eNOS in HUVEC-C. Total cell lysates were extracted from HUVEC-C exposed to $24 \mathrm{~h}$ of normal gravity (NG) or simulated microgravity (Clino) respectively, and subjected to immunoprecipitation using antibody against Cav-1. Precipitates were then subjected to Western blot analysis using antibodies against Cav-1 and total eNOS.

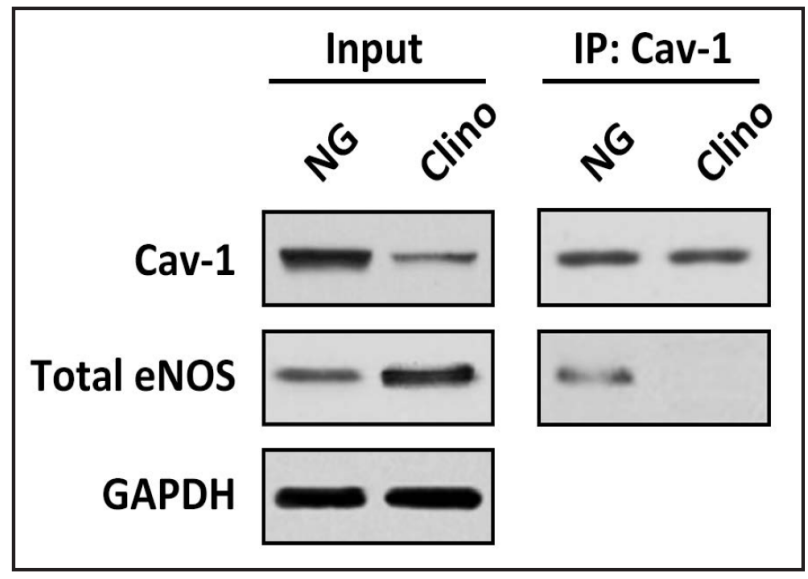

Microgravity disrupts Cav-1/eNOS protein interaction

To further investigate the protein interaction between Cav-1 and eNOS, we performed an immunoprecipitation assay (Fig. 3). As shown in the input samples, Cav-1 protein decreased while eNOS protein increased in whole cell lysates of the microgravity-treated cells, which was consistent with our earlier findings (Fig. 1C). Of note, the amount of Cav-1 protein in the immunoprecipitated samples from both treatment groups were comparable, likely due to the concentrating effect of immunoprecipitation using Cav-1 antibody. In contrast, Cav-1 associated eNOS proteins were greatly diminished in cells exposed to microgravity, as shown by decreased eNOS protein detected in the Cav-1-immunoprecipitated protein complexes (Fig. 3). This data suggested that in response to weightlessness, eNOS interaction with Cav-1 was extensively disrupted in HUVEC cells.

Cav-1 is critically involved in angiogenic response of endothelial cell in microgravity

The interaction between Cav-1 and eNOS inhibits eNOS enzyme activation [16, 28, 27] that potentially mediates angiogenic response of cells in microgravity [29-31]. We thus decided to assess the roles of Cav-1 in angiogenesis of HUVEC cells exposed to microgravity. First, to genetically manipulate Cav-1 expression in HUVEC cells, we established the specific Cav-1 gene knockdown and overexpression models (Fig. 4). A pool of siRNA oligos that target human Cav-1 was transfected into HUVEC cells 24 hours before the treatments for normal gravity or simulated microgravity. The scrambled oligos were used as control. As shown in Fig. 4A and 4B, comparing to control knockdown cells receiving normal gravity 
Fig. 4. Effects of shRNA knockdown and overexpression on Cav-1 levels in HUVEC-C. (A and B) mRNA (A) and protein (B) levels of Cav-1 were examined in HUVEC-C transfected with control shRNA following exposure to $24 \mathrm{~h}$ of normal gravity (Control+NG) or simulated microgravity (Control+Clino), and Cav-1 shRNA following exposure to $24 \mathrm{~h}$ of normal gravity (shR$\mathrm{NA}+\mathrm{NG}$ ). (C and D) mRNA (A) and protein (B) levels of Cav-1 were examined in HUVEC-C transfected with empty plasmid control following exposure to $24 \mathrm{~h}$ of normal gravity (Control+NG) or simulated microgravity (Control+Clino), and plasmid overexpressing Cav-1 following exposure to $24 \mathrm{~h}$ of simulated microgravity (OE+Clino). mRNA levels were normalized to $G A P D H$, and plotted as percentage relative to respective Control+NG groups. Western blots were quantified by densitometry analysis. Values are mean \pm SEM from three independent experiments. $* \mathrm{P}<$ 0.05 compared to respective Control+NG, n1 not significant compared to Control+Clino, n2 not significant compared to Control+NG.

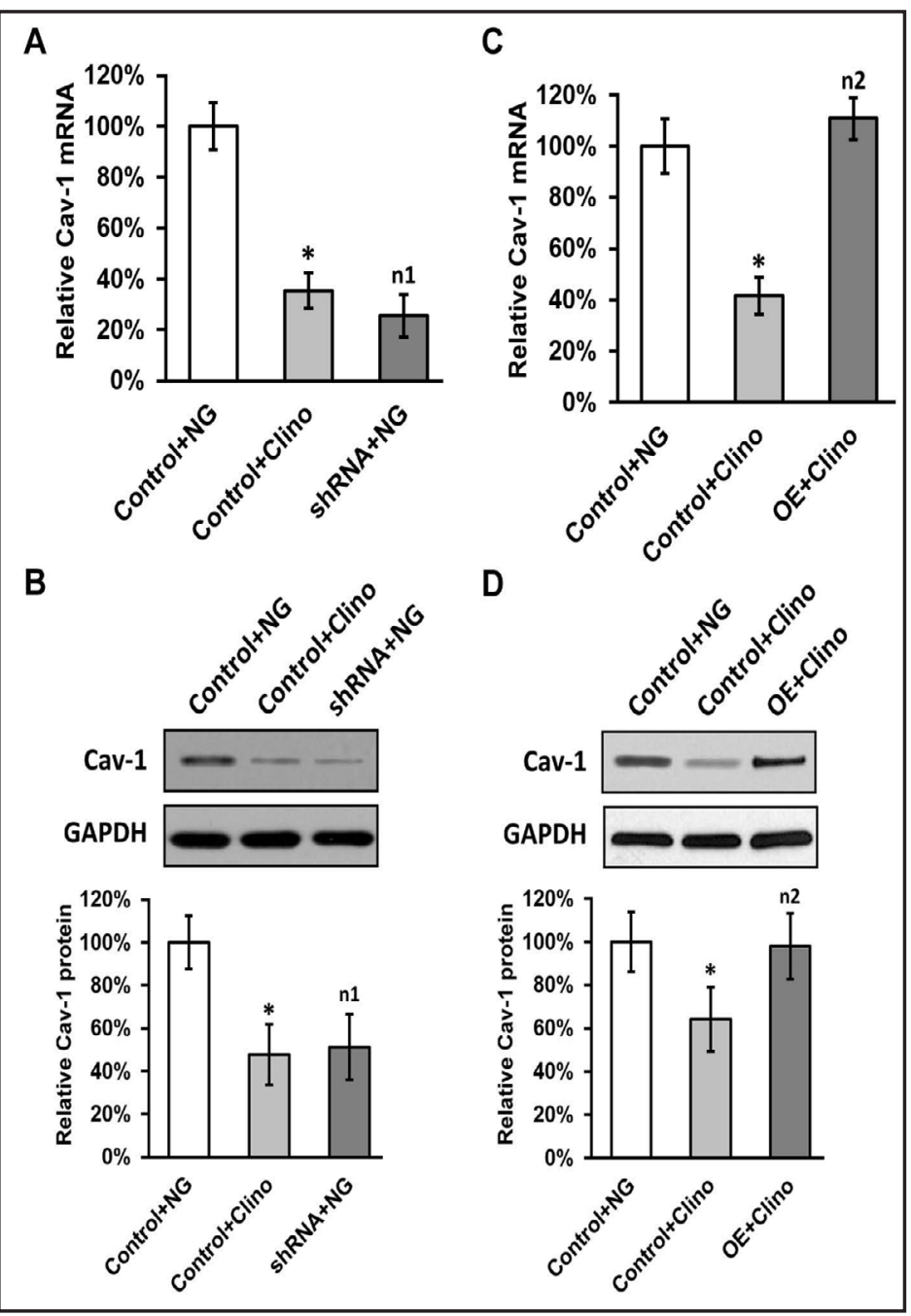

treatment (control+NG), the levels of Cav-1 transcript and protein were both reduced in siRNA-knockdown cells (siRNA+NG), confirming the feasibility of the loss-of-function strategy. Similarly, empty vector or Cav-1 overexpression plasmids were transfected into HUVEC cells followed by the clinorotation. The success of the gain-of-the function approach was supported by the evidence that the decreased Cav-1 expression (mRNA and protein) in microgravity-treated cells (Control+Clino) were rescued by the ectopic expression of Cav-1 (OE+Clino) in HUVEC cells before the treatments (Fig. 4C and 4D).

We then used these models to study cell migration and tube formation, both of which are considered as important angiogenic index in vitro. Comparing to normal gravity (Control+NG), HUVEC cells treated by microgravity (Control+Clino) have exhibited significantly increased capacities in both cell migration and tube formation assays (Fig. 5 and 6). Notably, these effects were closely mimicked in cells of normal gravity when Cav-1 expression was silenced (siRNA+NG in Fig. 7A and 7B). On the other hand, when Cav-1 expression was restored in cells exposed to microgravity (OE+Clino in Fig. 8A and 8B), the angiogenic potential, including enhanced cell migration in the scratch assay and the increased activity of the tube formation, was normalized to the similar levels of cells in the normal gravity group (Control+NG), both of which were less than the group of cells stimulated by microgravity alone (Control+Clino). These data strongly argued that the decrease of Cav-1 expression was both necessary and sufficient for the promotion of angiogenic responses of simulated weightlessness in endothelial cells. 
Fig. 5. Effects of Cav-1 shRNA knockdown on migration and tube formation of HUVEC-C. Representative images of cell migration (A) and tube formation (B) assays from HUVEC-C transfected with control shRNA following exposure to 24 $\mathrm{h}$ of normal gravity (Control+NG) or simulated microgravity (Control+Clino), and Cav-1 shRNA following exposure to $24 \mathrm{~h}$ of normal gravity (shRNA+NG).

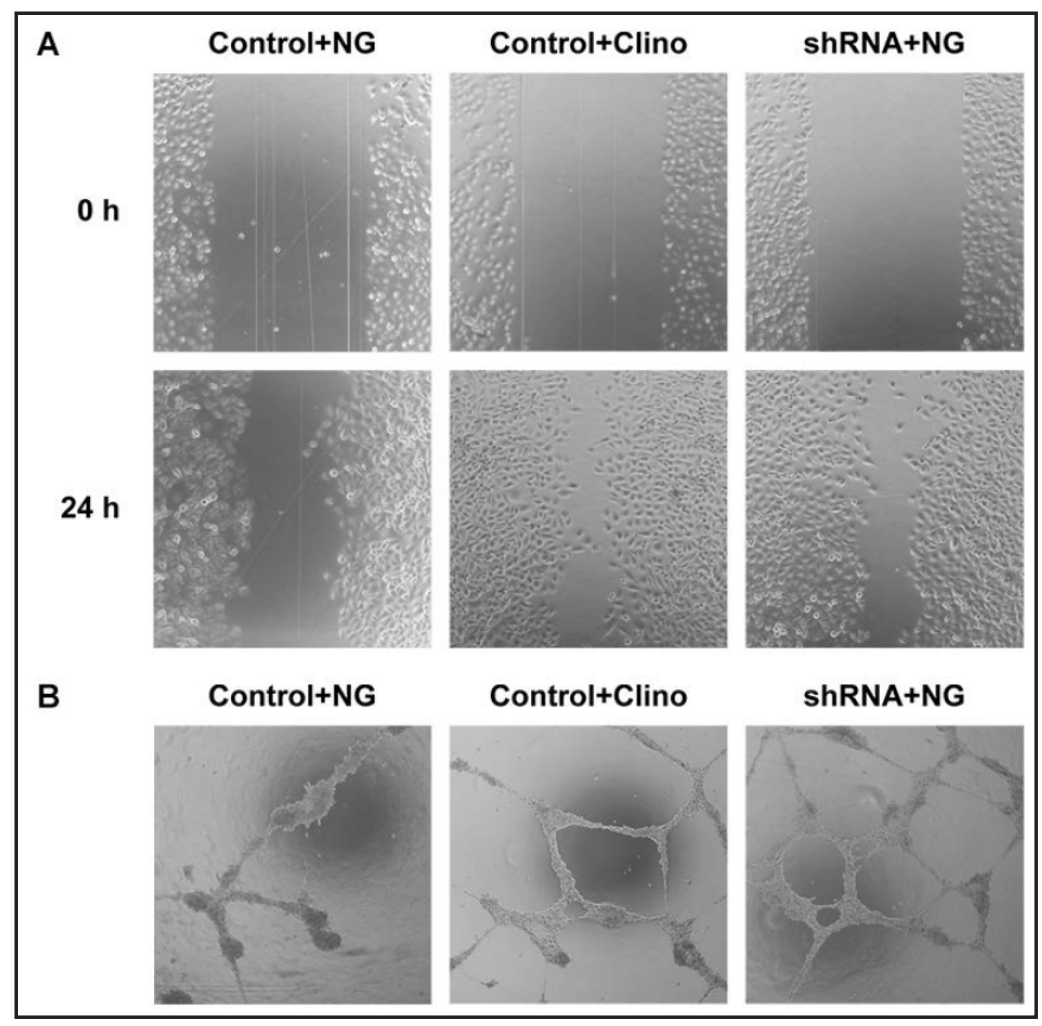

Fig. 6. Effects of Cav-1 overexpression on migration and tube formation of HUVEC-C. Representative images of cell migration (A) and tube formation (B) assays from HUVEC-C transfected with empty plasmid control following exposure to $24 \mathrm{~h}$ of normal gravity (Control+NG) or simulated microgravity (Control+Clino), and plasmid overexpressing Cav1 following exposure to $24 \mathrm{~h}$ of simulated microgravity (OE+Clino).

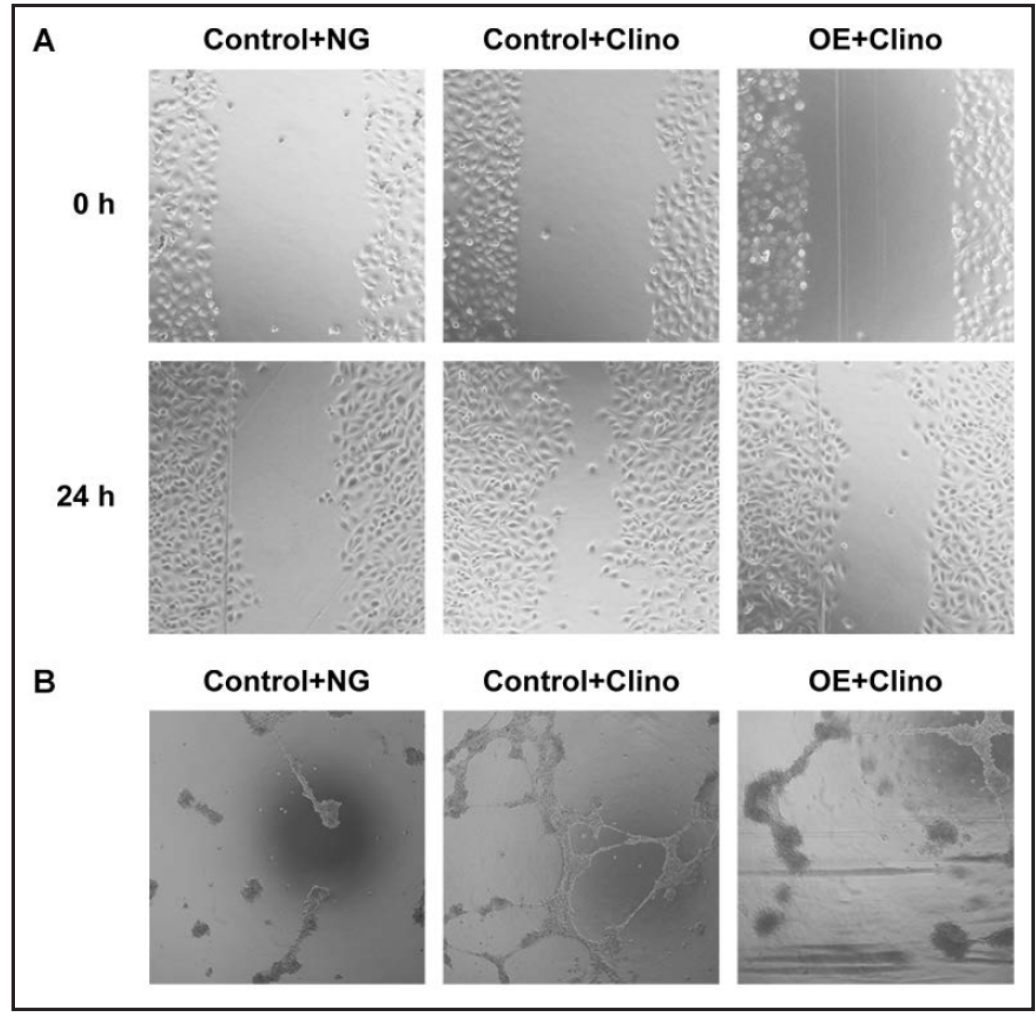

\section{Discussion}

The physiological changes in the human body during spaceflight contain an amazingly multitude of responses from molecular to tissue levels. For instance, the expressions of key surface receptors and appropriate regulation of signal molecules in primary human 


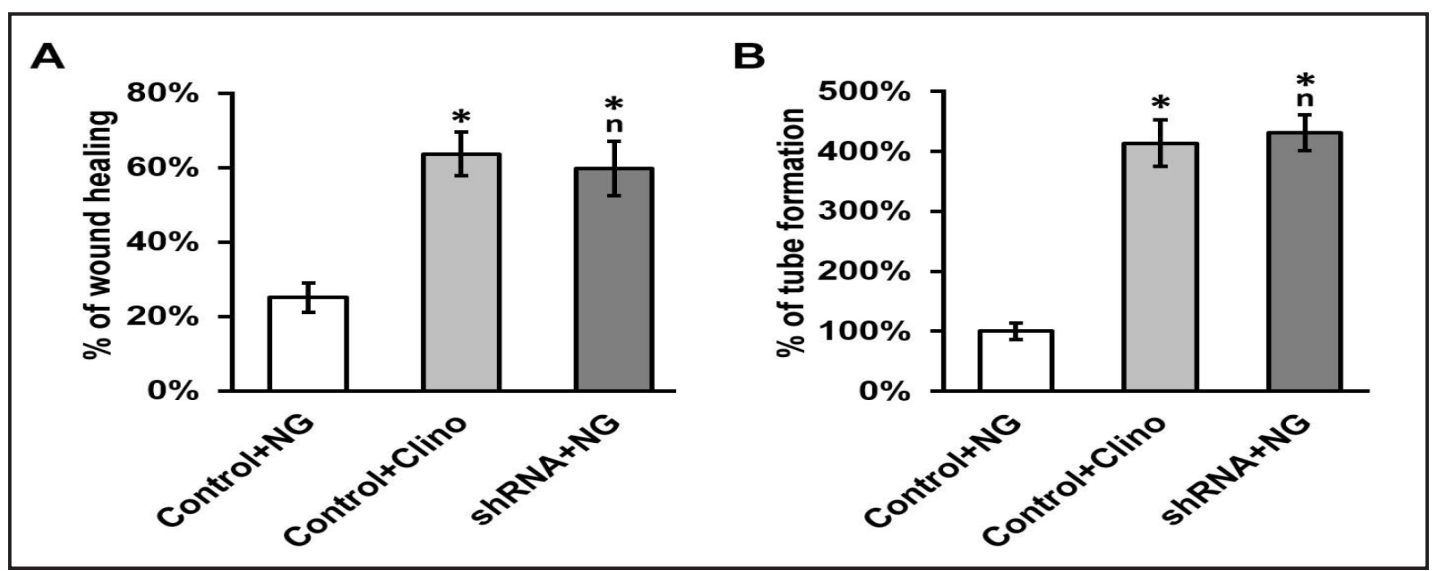

Fig. 7. Effects of Cav-1 shRNA knockdown on migration and tube formation of HUVEC-C. Percentage of cell migration (A) and tube formation (B) were examined in HUVEC-C transfected with control shRNA following exposure to $24 \mathrm{~h}$ of normal gravity (Control+NG) or simulated microgravity (Control+Clino), and Cav-1 shRNA following exposure to $24 \mathrm{~h}$ of normal gravity (shRNA+NG). Values are mean $\pm \mathrm{SEM}$ from three independent experiments. ${ }^{*} \mathrm{P}<0.05$ compared to Control+NG, n not significant compared to Control+Clino.

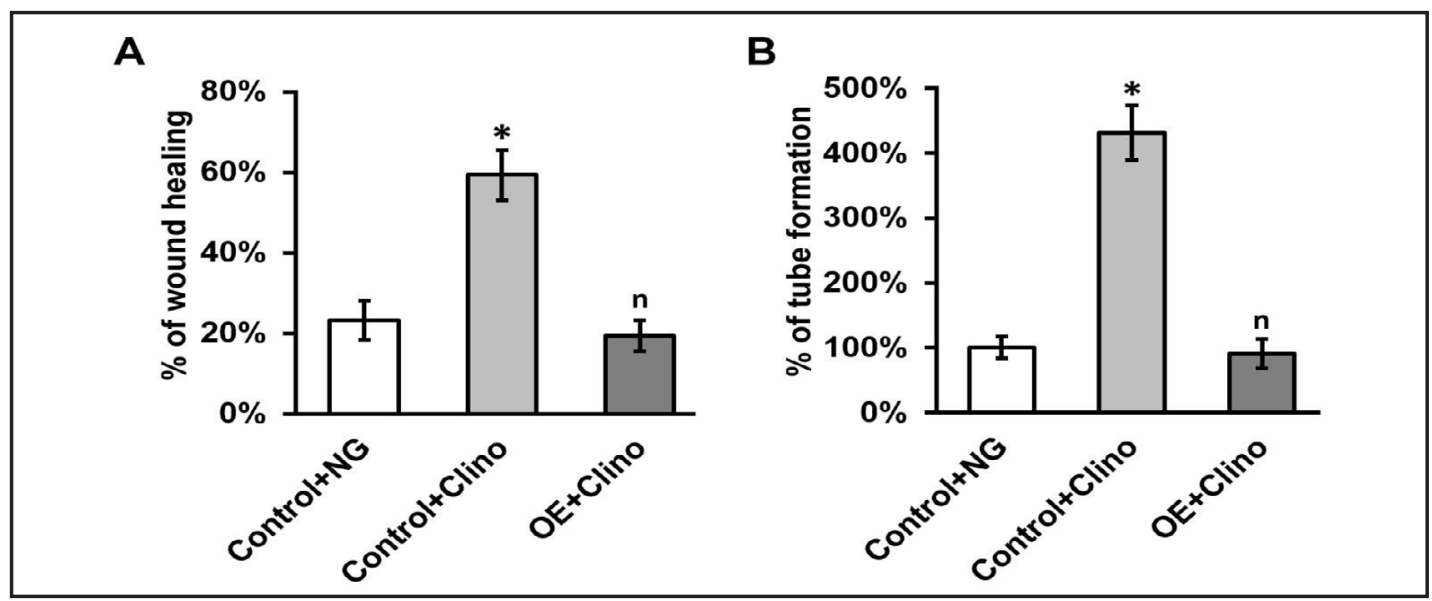

Fig. 8. Effects of Cav-1 overexpression on migration and tube formation of HUVEC-C. Percentage of cell migration (A) and tube formation (B) were examined in HUVEC-C transfected with empty plasmid control following exposure to $24 \mathrm{~h}$ of normal gravity (Control+NG) or simulated microgravity (Control+Clino), and plasmid overexpressing Cav-1 following exposure to $24 \mathrm{~h}$ of simulated microgravity (OE+Clino). Values are mean \pm SEM from three independent experiments. * $\mathrm{P}<0.05$ compared either Control $+\mathrm{NG}$ or $\mathrm{OE}+\mathrm{Clino}, \mathrm{n}$ not significant compared to Control+NG.

T lymphocytes was found to be altered during parabolic flight and clinostat experiments [32]. Our research on endothelial cells treated by simulated weightlessness highlights an important molecular player-Cav-1, during angiogenic response to microgravity. The expression of Cav-1 was decreased in HUVEC cells exposed to microgravity, unleashing the interaction with eNOS. Comparing to normal gravity, the disrupted eNOS association with Cav-1 resulted in the enhanced total protein and phosphorylation levels of eNOS, leading to induced angiogenic responses in simulated weightlessness. Importantly, from the genetic manipulation of Cav-1, our data demonstrated that the down-regulation of Cav-1 was a prerequisite step for promoting angiogenesis in endothelial cell responding to microgravity.

Cav-1 is a major resident protein in caveolae that may function as a mechanosensor in vascular cells for hydrodynamic adaptations, such as shear stress, blood flow, fluid pressure, etc. It was recently reported to be involved in regulating the proliferation of pulmonary 
microvascular endothelial cells in hepatopulmonary syndrome [33]. It has thus been studied particularly in microgravity-associated cellular responses $[21,29,30,31$. The changes of Cav-1 expression have been documented in several models. Consistent with our finding that microgravity reduced Cav-1 expression in HUVEC cells, it was recently found that the quantity of caveolae was reduced and their integrity was compromised in cells exposed to simulated weightlessness under transmission electron microscope [21]. In addition, in a rat head-down tail-up model of microgravity, Cav-1 protein was found to be lower in the middle cerebral artery [34]. On the other hand, the up-regulation of Cav-1 by microgravity has also been observed in thyroid [35] and in mechanical unloading [36], suggesting that tissue- and/ or context-specific regulation of Cav-1 by microgravity could be involved.

A variety of lipid-modified proteins trafficking through caveolae can interact with Cav-1, including eNOS, whose function is critical for angiogenesis [25, 27]. The functional significance of Cav-1/eNOS protein interaction has been extensively explored. First, co-transfection of Cav-1 and eNOS inhibits nitric oxide production, and mutants that specifically lack Cav-1/eNOS association restore eNOS activity $[16,26]$. Second, calciumdependent calmodulin disrupts the interaction by reciprocal binding with eNOS, leading to enzyme activation [28]. Finally, the physiologic relevance of Cav-1 effects on eNOS was suggested by Cav-1 null mouse models [25], in which vascular phenotypes were derived from the hyperactivation of eNOS. Following this rationale, our data that the Cav-1/eNOS association decreased, along with the stimulated eNOS expression, strongly suggested simulated weightlessness could activate eNOS signaling pathway in endothelial cells. Such a notion has been further supported by our findings that an increase in eNOS phosphorylation associated with enzyme activation was observed in microgravity-treated cells [17]. Several studies have observed similar induction in the expression and the phosphorylation of eNOS by microgravity in endothelial cells. In contrast, a previous study found no change in eNOS phosphorylation, even though the production of NO was greatly enhanced by microgravity in HUVEC cells [29]. Different mechanisms underlying eNOS activation therefore could contribute to the distinct observations that might be derived from the variations of the models used to simulate microgravity conditions. The data presented here provides a novel mechanistic insight that involves Cav-1/eNOS complex dissociation. Forcing ectopic expression of Cav-1 in cells treated with microgravity blocked the angiogenic response as expected. On the other hand, angiogenesis was induced in cells with normal gravity when Cav-1 was reduced by siRNA knockdown (Fig. 7). Thus, it is clear that the decoupling of Cav1 from eNOS association plays a central role for activating eNOS in endothelial cells treated with microgravity. Moreover, other Cav-1-mediated mechanisms may also be involved. For instance, it was found that the increased phosphorylation of Tyr-14 on Cav-1 protein could be responsible for eNOS activation [29]. On the other hand, the conventional PI3K/Aktstimulated eNOS phosphorylation cascades have been reported in HUVEC cells treated with microgravity [21], which could also play a synergistic role with Cav-1 in stimulating eNOS function.

NO-dependent functions in endothelial cells are known to play important roles in microgravity-associated cardiovascular complications. In hindlimb unloading animal model, a chronic model of simulated microgravity, NO production in the blood vessel was found to be elevated $[37,38]$. Interestingly, the expression of eNOS is modulated differently depending on the location and type of the blood vessels [38], suggesting differential adaptations in response to microgravity that could be sensitive to the environment. The eNOS-dependent NO production mainly promotes vasodilatory tones and blood flow [13]. Thus, up-regulation of eNOS/NO system in microgravity could contribute to cardiovascular deconditioning to microgravity during spaceflight. For instance, contractile hyporesponses in pulmonary arteries from animals in simulated microgravity could be caused by the enhanced eNOS activity [39]. Significantly, our studies using cell models of simulated microgravity have suggested that the increased expression and phosphorylation of eNOS may be responsible for an enhanced angiogenic response in HUVEC cells stimulated by microgravity [21]. It could have several implications on both microgravity-associated pathophysiology and vascular 
research. First, non-reparative angiogenesis-based neovascularization is often associated with detrimental effects in microvascular networks to induce unstable and immature vessels [40]. Retinopathy-like impairment has also been found in human during spaceflights [41]. In addition, eNOS-produced NO could be a potent pro-inflammatory factor, especially when combined with oxidative stress $[42,43]$. Stimulated by microgravity, eNOS can be directly involved in the inflammation responses in the blood vessels [36]. Second, microgravitystimulated angiogenesis establishes a feasible and functional protocol for studying tissue engineering $[44,10]$. It has been successfully developed for inducing perfusion in three-dimensional in vitro organized tissues, such as in the liver and bones [44, 10, 45]. Microgravity-stimulated tube formation presented a useful model for studying the essential signaling, such as Cav-1 dissociation with eNOS in our present studies, during vascular conformation in vivo. Given that eNOS signaling pathway was widely reported to be involved in angiogenesis, our current data also suggested that the modulation of Cav-1/caveolae may be relevant to neovascularization in other conditions such as malignancy. The potential mechanisms that initiate, mediate and induce Cav-1 dissociation with eNOS in microgravity may require further investigation in the future. It will also be worth dissecting if other Cav$1 /$ caveolae associated signaling molecules play any potential functions during simulated weightlessness in the endothelium or other relevant cell types. Affecting caveolae through mechano-transduction, microgravity may regulate other isoforms of caveolin, such as Cav-2 which is also expressed abundantly in the endothelial cells, and in turn play important roles in angiogenesis [46]. In order to obtain a detailed investigation on caveolae deformation stimulated by microgravity, it would be interesting to assess these caveolin isoforms as well.

\section{Conclusion}

Simulated weightlessness promotes eNOS-mediated angiogenesis such as cell migration and tube formation in vitro. The expression of Cav-1 decreased while eNOS expression increased in HUVEC cells exposed to microgravity. Importantly, Cav-1/eNOS interactions were greatly diminished in microgravity, thus leading to eNOS-phosphorylation-associated enzyme activation. Our results demonstrate the requirement of Cav-1-assoicated signaling in stimulated angiogenesis by microgravity, suggesting that Cav-1 is a critical mediator in simulated weightlessness on endothelium-dependent angiogenesis.

\section{Acknowledgments}

This work was supported by the National Natural Science Foundation of China (81301681, 81372130 and 81200902), the Army Youth Innovation \& Incubator Foundation (13QNP123). The funders had no role in study design, data collection and analysis, decision to publish, or preparation of the manuscript. We are grateful to Dr. Jing Sun, Dr. Huan Liu and Dr. Jun Hu for their valuable suggestions on this work. The authors have no conflicts to disclose.

\section{Disclosure Statement}

None to declare.

\section{References}

1 Buckey JC Jr, Lane LD, Levine BD, Watenpaugh DE, Wright SJ, Moore WE, Gaffney FA, Blomqvist CG: Orthostatic intolerance after spaceflight. J Appl Physiol (1985) 1996;81:7-18.

2 Lang T, LeBlanc A, Evans H, Lu Y, Genant H, Yu A: Cortical and trabecular bone mineral loss from the spine and hip in long-duration spaceflight. J Bone Miner Res 2004;19:1006-1012. 


\section{Cellular Physiology Cell Physiol Biochem 2016;38:502-513 \begin{tabular}{l|l|l|l} 
DOI: 10.1159/000438646 & (C) 2016 The Author(s). Published by S. Karger AG, Basel \\
www.karger.com/cpb
\end{tabular}}

Shi et al.: Endothelial Functions in Altered Gravitational Environments

3 Fitts RH, Riley DR, Widrick JJ: Physiology of a microgravity environment invited review: microgravity and skeletal muscle. J Appl Physiol (1985) 2000;89:823-839.

4 Levine BD, Zuckerman JH, Pawelczyk JA, Blomqvist CG: Cardiac atrophy after bed-rest deconditioning: a nonneural mechanism for orthostatic intolerance. Circulation 1997;96:517-525.

5 Lewis ML, Reynolds JL, Cubano LA, Hatton JP, Lawless BD, Piepmeier EH: Spaceflight alters microtubules and increases apoptosis in human lymphocytes (Jurkat). Faseb J 1998;12:1007-1018.

$6 \quad$ Zhang LF: Vascular adaptation to microgravity: what have we learned? J Appl Physiol (1985) 2001;91:2415-2430.

7 Fritsch-Yelle JM, Charles JB, Jones MM, Wood ML: Microgravity decreases heart rate and arterial pressure in humans. J Appl Physiol (1985) 1996;80:910-914.

8 Convertino VA: Cardiovascular consequences of bed rest: effect on maximal oxygen uptake. Med Sci Sports Exerc 1997;29:191-196.

9 Carlsson SIM, Bertilaccio MTS, Ballabio E, Maier JAM: Endothelial stress by gravitational unloading: effects on cell growth and cytoskeletal organization. Biochim Biophys Acta 2003;1642:173-179.

10 Infanger M, Kossmehl P, Shakibaei M, Baatout S, Witzing A, Grosse J, Bauer J, Cogoli A, Faramarzi S, Derradji H, Neefs M, Paul M, Grimm D: Induction of three-dimensional assembly and increase in apoptosis of human endothelial cells by simulated microgravity: impact of vascular endothelial growth factor. Apoptosis 2006;11:749-764.

11 Semenza GL: Vasculogenesis, angiogenesis, and arteriogenesis: mechanisms of blood vessel formation and remodeling. J Cell Biochem 2007;102:840-847.

12 Carmeliet P: Mechanisms of angiogenesis and arteriogenesis. Nat Med 2000;6:389-395.

13 Huang PL: eNOS, metabolic syndrome and cardiovascular disease. Trends Endocrinol Metab 2009;20:295302.

14 Murohara T, Asahara T, Silver M, Bauters C, Masuda H, Kalka C, Kearney M, Chen D, Symes JF, Fishman MC, Huang PL, Isner JM: Nitric oxide synthase modulates angiogenesis in response to tissue ischemia. J Clin Invest 1998;101:2567-2578.

15 Papapetropoulos A, Garcia-Cardena G, Madri JA, Sessa WC: Nitric oxide production contributes to the angiogenic properties of vascular endothelial growth factor in human endothelial cells. J Clin Invest 1997;100:3131-3139.

16 Garcia-Cardena G, Martasek P, Masters BS, Skidd PM, Couet J, Li S, Lisanti MP, Sessa WC: Dissecting the interaction between nitric oxide synthase (NOS) and caveolin. Functional significance of the nos caveolin binding domain in vivo. J Biol Chem 1997;272:25437-25440.

17 Fleming I, Busse R: Molecular mechanisms involved in the regulation of the endothelial nitric oxide synthase. Am J Physiol Regul Integr Comp Physiol 2003;284:R1-12.

18 Garcia-Cardena G, Fan R, Shah V, Sorrentino R, Cirino G, Papapetropoulos A, Sessa WC: Dynamic activation of endothelial nitric oxide synthase by Hsp90. Nature 1998;392:821-824.

19 Shaul PW, Smart EJ, Robinson LJ, German Z, Yuhanna IS, Ying Y, Anderson RG, Michel T: Acylation targets emdothelial nitric-oxide synthase to plasmalemmal caveolae. J Biol Chem 1996;271:6518-6522.

20 Arnaoutova I, Kleinman HK: In vitro angiogenesis: endothelial cell tube formation on gelled basement membrane extract. Nat Protoc 2010;5:628-635.

21 Shi F, Wang Y-C, Zhao T-Z, Zhang S, Du T-Y, Yang C-B, Li Y-H, Sun X-Q: Effects of simulated microgravity on human umbilical vein endothelial cell angiogenesis and role of the PI3K-Akt-eNOS signal pathway. PloS one 2012;7:e40365.

22 Rothberg KG, Heuser JE, Donzell WC, Ying YS, Glenney JR, Anderson RG: Caveolin, a protein component of caveolae membrane coats. Cell 1992;68:673-682.

23 Sargiacomo M, Sudol M, Tang Z, Lisanti MP: Signal transducing molecules and glycosylphosphatidylinositol-linked proteins form a caveolin-rich insoluble complex in MDCK cells. J Cell Biol 1993;122:789-807.

24 Song KS, Li S, Okamoto T, Quilliam LA, Sargiacomo M, Lisanti MP: Co-purification and direct interaction of Ras with caveolin, an integral membrane protein of caveolae microdomains. Detergent-free purification of caveolae microdomains. J Biol Chem 1996;271:9690-9697.

25 Razani B, Engelman JA, Wang XB, Schubert W, Zhang XL, Marks CB, Macaluso F, Russell RG, Li M, Pestell RG, Di Vizio D, Hou H, Jr., Kneitz B, Lagaud G, Christ GJ, Edelmann W, Lisanti MP: Caveolin-1 null mice are viable but show evidence of hyperproliferative and vascular abnormalities. J Biol Chem 2001;276:38121-38138. 


\section{Cellular Physiology Cell Physiol Biochem 2016;38:502-513

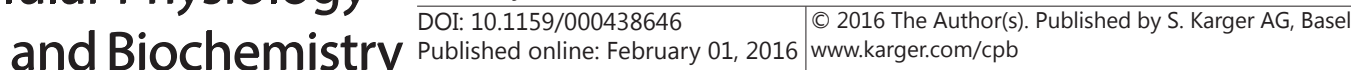

Shi et al.: Endothelial Functions in Altered Gravitational Environments

26 Couet J, Li S, Okamoto T, Ikezu T, Lisanti MP: Identification of peptide and protein ligands for the caveolinscaffolding domain. Implications for the interaction of caveolin with caveolae-associated proteins. J Biol Chem 1997;272:6525-6533.

27 Ju H, Zou R, Venema VJ, Venema RC: Direct interaction of endothelial nitric-oxide synthase and caveolin-1 inhibits synthase activity. J Biol Chem 1997;272:18522-18525.

28 Michel JB, Feron O, Sacks D, Michel T: Reciprocal regulation of endothelial nitric-oxide synthase by Ca2+calmodulin and caveolin. J Biol Chem 1997;272:15583-15586.

29 Spisni E, Toni M, Strillacci A, Galleri G, Santi S, Griffoni C, Tomasi V: Caveolae and caveolae constituents in mechanosensing: effect of modeled microgravity on cultured human endothelial cells. Cell Biochem Biophys 2006;46:155-164.

30 Maier JA, Cialdai F, Monici M, Morbidelli L: The impact of microgravity and hypergravity on endothelial cells. Biomed Res Int 2015;2015:434803.

31 Wang Z, Bai Y, Yu J, Liu H, Cheng Y, Liu Y, Xie X, Ma J, Bao J: Caveolae regulate vasoconstriction of conduit arteries to angiotensin II in hindlimb unweighted rats. J Physiol 2015;593:4561-4574.

32 Tauber S, Hauschild S, Paulsen K, Gutewort A, Raig C, Hurlimann E, Biskup J, Philpot C, Lier H, Engelmann F, Pantaleo A, Cogoli A, Pippia P, Layer LE, Thiel CS, Ullrich O: Signal transduction in primary human T lymphocytes in altered gravity during parabolic flight and clinostat experiments. Cell Physiol Biochem 2015;35:1034-1051.

33 Zeng J, Chen L, Chen B, Lu K, Belguise K, Wang X, Yi B: MicroRNA-199a-5p Regulates the Proliferation of Pulmonary Microvascular Endothelial Cells in Hepatopulmonary Syndrome. Cell Physiol Biochem 2015;37:1289-1300.

34 Prisby RD, Wilkerson MK, Sokoya EM, Bryan RM, Jr., Wilson E, Delp MD: Endothelium-dependent vasodilation of cerebral arteries is altered with simulated microgravity through nitric oxide synthase and EDHF mechanisms. J Appl Physiol (1985) 2006;101:348-353.

35 Masini MA, Albi E, Barmo C, Bonfiglio T, Bruni L, Canesi L, Cataldi S, Curcio F, D'Amora M, Ferri I, Goto K, Kawano F, Lazzarini R, Loreti E, Nakai N, Ohira T, Ohira Y, Palmero S, Prato P, Ricci F, Scarabelli L, Shibaguchi T, Spelat R, Strollo F, Ambesi-Impiombato FS: The impact of long-term exposure to space environment on adult mammalian organisms: a study on mouse thyroid and testis. PloS one 2012;7:e35418.

36 Grenon SM, Jeanne M, Aguado-Zuniga J, Conte MS, Hughes-Fulford M: Effects of gravitational mechanical unloading in endothelial cells: association between caveolins, inflammation and adhesion molecules. Sci Rep 2013;3:1494.

37 Vaziri ND, Ding Y, Sangha DS, Purdy RE: Upregulation of NOS by simulated microgravity, potential cause of orthostatic intolerance. J Appl Physiol (1985) 2000;89:338-344.

38 Ma J, Kahwaji CI, Ni Z, Vaziri ND, Purdy RE: Effects of simulated microgravity on arterial nitric oxide synthase and nitrate and nitrite content. J Appl Physiol (1985) 2003;94:83-92.

39 Nyhan D, Kim S, Dunbar S, Li D, Shoukas A, Berkowitz DE: Impaired pulmonary artery contractile responses in a rat model of microgravity: role of nitric oxide. J Appl Physiol (1985) 2002;92:33-40.

40 Carmeliet P: Angiogenesis in health and disease. Nat Med 2003;9:653-660.

41 Kergoat H, Durand MJ: Correlating increased ocular and systemic blood pressures with neuroretinal function. Aviat Space Envir Md 1996;67:1174-1178.

42 Forstermann U, Munzel T: Endothelial nitric oxide synthase in vascular disease: from marvel to menace. Circulation 2006;113:1708-1714.

43 Kim JH, Bugaj LJ, Oh YJ, Bivalacqua TJ, Ryoo S, Soucy KG, Santhanam L, Webb A, Camara A, Sikka G, Nyhan D, Shoukas AA, Ilies M, Christianson DW, Champion HC, Berkowitz DE: Arginase inhibition restores NOS coupling and reverses endothelial dysfunction and vascular stiffness in old rats. J Appl Physiol (1985) 2009;107:1249-1257.

44 Khaoustov VI, Darlington GJ, Soriano HE, Krishnan B, Risin D, Pellis NR, Yoffe B: Induction of threedimensional assembly of human liver cells by simulated microgravity. In Vitro Cell Dev-An 1999;35:501509.

45 Kirchen ME, O'Connor KM, Gruber HE, Sweeney JR, Fras IA, Stover SJ, Sarmiento A, Marshall GJ: Effects of microgravity on bone healing in a rat fibular osteotomy model. Clin Orthop Relat Res 1995:231-242.

46 Xie L, Frank PG, Lisanti MP, Sowa G: Endothelial cells isolated from caveolin-2 knockout mice display higher proliferation rate and cell cycle progression relative to their wild-type counterparts. Am J Physiol Cell Physiol 2010;298:C693-701. 